\title{
Stationary phase approximation in the ambient noise method revisited*
}

\author{
Zhongwen Zhan ${ }^{1}$ and Sidao $\mathrm{Ni}^{2,+}$ \\ ${ }^{1}$ Mengcheng National Geophysical Observatory, School of Earth and Space Sciences, \\ University of Science and Technology of China, Hefei 230026, China \\ ${ }^{2}$ Key Laboratory of Dynamic Geodesy, Institute of Geodesy and Geophysics, Chinese \\ Academy of Sciences, Wuhan 430077, China
}

\begin{abstract}
The method of extracting Green's function between stations from cross correlation has proven to be effective theoretically and experimentally. It has been widely applied to surface wave tomography of the crust and upmost mantle. However, there are still controversies about why this method works. Snieder employed stationary phase approximation in evaluating contribution to cross correlation function from scatterers in the whole space, and concluded that it is the constructive interference of waves emitted by the scatterers near the receiver line that leads to the emergence of Green's function. His derivation demonstrates that cross correlation function is just the convolution of noise power spectrum and the Green's function. However, his derivation ignores influence from the two stationary points at infinities, therefore it may fail when attenuation is absent. In order to obtain accurate noise-correlation function due to scatters over the whole space, we compute the total contribution with numerical integration in polar coordinates. Our numerical computation of cross correlation function indicates that the incomplete stationary phase approximation introduces remarkable errors to the cross correlation function, in both amplitude and phase, when the frequency is low with reasonable quality factor $Q$. Our results argue that the distance between stations has to be beyond several wavelengths in order to reduce the influence of this inaccuracy on the applications of ambient noise method, and only the station pairs whose distances are above several $(>5)$ wavelengths can be used.
\end{abstract}

Key words: ambient seismic noise; stationary phase approximation; Green's function

CLC number: P315.01 Document code: A

\section{Introduction}

In recent years, it is shown that the inter-receiver Green's function could be extracted from cross correlation of ambient noise recorded at the receivers. This method was first reported in ultrasonic experiments (Weaver and Lobkis, 2001, 2002) and then has been widely applied to crustal seismology to get high resolution surface wave tomography (Shapiro and Campillo, 2004; Shapiro et al., 2005). The scales of studied regions range from regional (Sabra et al., 2005; Kang and Shin, 2006; Yao et al., 2006; Villasenor et al., 2007; Cho et al., 2007) to continental (Yang et al., 2006). However, there are still a lot of controversies about why cross correlation of ambient seismic noise leads to

\footnotetext{
* Received 28 April 2010; accepted in revised form 14 August 2010; published 10 October 2010 .

^Corresponding author. e-mail: sdni@ustc.edu.cn

(C) The Seismological Society of China and Springer-Verlag Berlin Heidelberg 2010
}

Green's function. Some scientists argued that either equipartition of modes or perfect diffusivity of wave field is necessary (Weaver and Lobkis, 2001, 2002; Shapiro and Campillo, 2004; Shapiro et al., 2005), which is indeed the case in ultrasonic experiments, but might not be realistic for the ambient seismic noise (Snieder, 2004). Instead, a derivation based on time-reversal invariance (Derode et al., 2003a, b) seems to be more physically attractive when the wavefield is not diffuse or the medium is infinite, though attenuation will break this time-reversal invariance (Snieder, 2007). In an effort to overcome the theoretical difficulties mentioned above, Snieder (2004) took a nice mathematical approach involving stationary phase approximation for homogeneous elastic medium. His derivation relaxes the global requirement of equipartitioning of normal modes to the local requirement that the scattered waves propagate isotropically near the receivers. Moreover, Snieder (2007) generalized this idea to inhomogeneous attenu- 
ating acoustic waves, which is much closer to the situation of seismic waves in a realistic world. In these two papers, he demonstrated that the emergence of Green's function is a direct consequence of constructive interference only of those scattered waves that propagate along the line connecting receivers. When the scatters are not near the receiver line, their contribution to cross correlation seems to disappear if the stationary phase approximation is assumed.

However, we know that for a homogeneous medium, the sources located on any hyperbola with its foci at the two receivers, not only the receiver line, produce stable phase difference at the two receivers. So we would like to investigate whether the stationary phase approximation has been applied appropriately, in order to understand the physics of emergence of Green's function from ambient noise.

We first examine Snieder's derivation by integrating the contribution from scatters distributed on a line perpendicular to the receiver line. Following that, we compute the cross correlation function in polar coordinates and compare the difference of phase and amplitude between cross correlation and Green's function. Finally, we discuss inaccuracy of ambient noise method due to the deviation of cross correlation from the real Green's function.

The rest of this paper is divided into three parts. In section 2, we will analyze and formulate the problems, with and without the stationary phase approximation. And then in section 3, we will present and discuss the deviation of the approximate solution given by Snieder (2004) from the exact solution from numerical computation. Section 4 is the conclusion.

\section{Numerical integral of NCF}

At regional scale, numerous studies have shown that the Green's function extracted from ambient seismic noise is dominated by surface waves (e.g., Shapiro and Campillo, 2004; Shapiro et al., 2005; Sabra et al., 2005; Yao et al., 2006), though there are also some reports of body waves retrieved (e.g., Roux et al., 2005; Draganov et al., 2009; Zhan et al., 2010). In this paper, we only discuss the wave equation in a 2-D attenuating homogeneous medium. For brevity, the simplest case of scalar waves (acoustic waves) is chosen to illustrate our opinions. The geometry for our analysis is similar to that of Snieder (2004) as shown in Figure 1. The two receivers, $A$ and $B$, are separated by a distance of $R$ and located on the $x$ axis. The origin of our coordinate system is chosen at receiver $A$. The dashed line $L_{0}$ in Figure 1 separates the left and the right half-plane. The scatterers act as secondary sources of singly and multiply scattered waves. Scatterer numbered $s$ emits a signal $S_{s}(t)$ that is due to all the waves that impinge upon that scatterer. Apart from the scatterers, the medium's phase velocity $c$ and the quality factor $Q$ are assumed to be spatially constant. In this case, the Green's function $G\left(\boldsymbol{r}, \boldsymbol{r}_{0}\right)$ in the frequency domain, that is the displacement response at $\boldsymbol{r}$ to a force $\delta\left(\boldsymbol{r}-\boldsymbol{r}_{0}\right)$, is given by

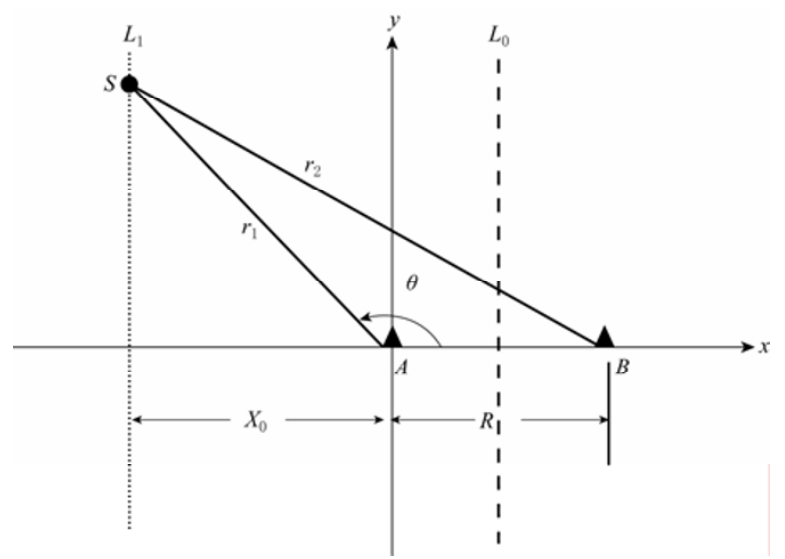

Figure 1 Definition of the geometric variables for our analysis. The black triangles denote the receivers $A$ and $B$. The dashed line $L_{0}$ is the perpendicular bisector of the segment $A B$. We compute integral $I(Y)$ numerically over the vertical dotted line $L_{1}, x=X_{0}$.

$$
G\left(\boldsymbol{r}, \boldsymbol{r}_{0}\right)=\sqrt{\frac{2}{\pi k R}} \mathrm{e}^{\mathrm{i}(k R+\pi / 4)} \mathrm{e}^{-R / 2 L},
$$

where $k$ is the wavenumber, $R$ is the distance between $r$ and $\boldsymbol{r}_{0}$, and $L=Q / k$ is the attenuation length of the medium (Snieder, 2004). Equation (1) is a far-field approximation of the Hankel function in the 2-D surface wave Green's function. All expressions in this work are formulated in the frequency domain. The wave field at the two receivers $A$ and $B$ can be written as a superposition of the waves radiated by the scatterers:

$$
u_{A, B}(\omega)=\sum_{s} G\left(\boldsymbol{r}_{A, B}, \boldsymbol{r}_{s}\right) S_{s}(\omega)
$$

where $\omega$ is the angular frequency and $S_{s}(\omega)$ is the Fourier transform of $S_{s}(t)$, that is the frequency spectrum of scatterer $s$. Equation (2) implies that the waves emitted by scatterer $s$ are isotropic, i.e., independent of directions. The summation is over all the scatterers on the plane. As shown by Snieder (2004), the scatterers distributed on the left half-plane (to the left of the dashed line $L_{0}$ in 
Figure 1) contribute to the causal Green's function $G\left(\boldsymbol{r}_{B}, \boldsymbol{r}_{A}\right)$, while the scatterers on the right half-plane give the acausal Green's function $G^{*}\left(\boldsymbol{r}_{B}, \boldsymbol{r}_{A}\right)$. Hereafter, only the scatterers on the left half-plane are considered and the summation is restricted to the left half-plane.

The cross correlation function between displacement records at the two receivers can be written as

$$
\begin{gathered}
C(\omega)=u_{A}^{*}(\omega) u_{B}(\omega)= \\
\sum_{s, s^{\prime}} G^{*}\left(\boldsymbol{r}_{A}, \boldsymbol{r}_{s}\right) G\left(\boldsymbol{r}_{B}, \boldsymbol{r}_{s^{\prime}}\right) S_{s}^{*}(\omega) S_{s^{\prime}}(\omega) .
\end{gathered}
$$

Following Snieder (2004), the double sum $\sum_{s, s^{\prime}}$ could be split into a sum over diagonal terms $\sum_{s=s^{\prime}}$ and a sum $\sum_{s \neq s^{\prime}}$ over cross terms. The sum of cross terms could be ignored by averaging over sufficient time/sources (Snieder, 2004). So

$$
C(\omega)=\sum_{s} G^{*}\left(\boldsymbol{r}_{A}, \boldsymbol{r}_{s}\right) G\left(\boldsymbol{r}_{B}, \boldsymbol{r}_{s}\right)\left|S_{s}(\omega)\right|^{2} .
$$

As there are many scatterers per wavelength, the resulting sum $\sum_{s}(\cdots)$ could be replaced by a surface integral

$$
\begin{aligned}
& C(\omega) \int_{\Sigma} G^{*}\left(\boldsymbol{r}_{A}, \boldsymbol{r}\right) G\left(\boldsymbol{r}_{B}, \boldsymbol{r}\right)|S(\omega)|^{2} n \mathrm{~d} x \mathrm{~d} y= \\
& \frac{2}{\pi k} \int_{\Sigma} \sqrt{\frac{1}{r_{1} r_{2}}} \mathrm{e}^{\mathrm{i} k\left(r_{2}-r_{1}\right)} \mathrm{e}^{-\left(r_{1}+r_{2}\right) / 2 L}|S(\omega)|^{2} n \mathrm{~d} x \mathrm{~d} y,
\end{aligned}
$$

where $n$ is the scatterer density per unit surface area and the $\sum$ stands for the left half-plane. The geometry of variables $r_{1}$ and $r_{2}$ is shown in Figure 1. Equation (5) is just the equation (9) in Snieder (2004), but with attenuation taken into account.

\subsection{Validity of stationary phase approximation}

There are different ways to solve the integral in equation (5). Snieder (2004) evaluated the integral over the transverse coordinate $y$ in the stationary phase approximation, and then evaluated the simpler integral over the coordinate $x$. These steps lead to a simple but important result:

$$
C(\omega)=\pi n L c \frac{G\left(\boldsymbol{r}_{B}, \boldsymbol{r}_{A}\right)}{\mathrm{i} \omega} \overline{|S(\omega)|^{2}},
$$

where $\overline{|S(\omega)|^{2}}$ is the average power spectrum of scatterers. This equation means that if we know $\overline{|S(\omega)|^{2}}$ we can extract the Green's function $G\left(\boldsymbol{r}_{B}, \boldsymbol{r}_{A}\right)$ between $A$ and $B$ from the cross correlation function $C(\omega)$.

Obviously, the point $y=0$ is the stationary point of the phase term $\left(k\left(r_{2}-r_{1}\right)\right)$ in equation (5) with the transverse coordinate $y$ as variable for given $x=x_{0}\left(L_{1}\right)$. This is the only stationary point that Snieder considered in his derivation. However, the phase term approaches zero at $y= \pm \infty$ and these stationary points should be taken into account. Though the contribution from $\pm \infty$ vanishes for attenuating media, we do not know the exact behavior of the competition between weaker contribution due to attenuation at longer propagation distances and stronger contribution due to constructive interference when $y$ becomes very large. Therefore, we conduct numerical integration along the transverse line $L_{1}$ for different quality factor $Q$ and frequency to investigate the behavior.

We calculate the integral (equation (5)) on the line $L_{1}$ with $X_{0}=R=100 \mathrm{~km}$, with Gaussian quadrature because of its higher efficiency. In Figure 2, we present the results for two $Q$ factors (300 and 3000$)$ and three frequencies $(0.2 \mathrm{~Hz}, 0.5 \mathrm{~Hz}, 2 \mathrm{~Hz})$. The case $Q=3000$ describes an unrealistic weak attenuation for the Earth, while $Q=300$ is a reasonable value for shield regions (Cong and Mitchell, 1998).

$$
\begin{gathered}
I(Y)= \\
\frac{2}{\pi k} \int_{-Y}^{Y}\left\{\sqrt{\frac{1}{r_{1} r_{2}}} \mathrm{e}^{\mathrm{i} k\left(r_{2}-r_{1}\right)} \mathrm{e}^{-\left(r_{1}+r_{2}\right) / 2 L}|S(\omega)|^{2} n\right\}_{x=X_{0}} \mathrm{~d} y
\end{gathered}
$$

In each panel of Figure 2, the solid lines and dashed lines are integrals $I(Y)$ for numerical computation and stationary phase approximation, respectively (red for real and blue for imaginary). For $Y$ close to zero, the two approaches match very well, suggesting that the stationary phase approximation works near the stationary point $Y=0$. At high frequency $(\geq 2 \mathrm{~Hz})$, the match is even good for large $Y$. However, at lower frequencies $(0.2 \mathrm{~Hz}$ and $0.5 \mathrm{~Hz}$ ), when $Y$ becomes larger and larger, curves from the two approaches are different from each other, so do their limits.

Based on Figure 2, we can conclude that it is inappropriate to evaluate the integral over the transverse coordinate $y$ by the stationary phase approximation, either for realistic $(Q=300)$ or low-attenuation media. So in the next section, we compute the integral numerically over the whole plane and discuss the errors incorporated by stationary phase approximation.

\subsection{Numerical evaluation in polar coordinate}

An alternative way is to compute the integral in equation (5) numerically, without using any approximation. As there is singularity at receiver $A$ because of $r_{1}$ at the denominator, we need to transform equation (5) to 

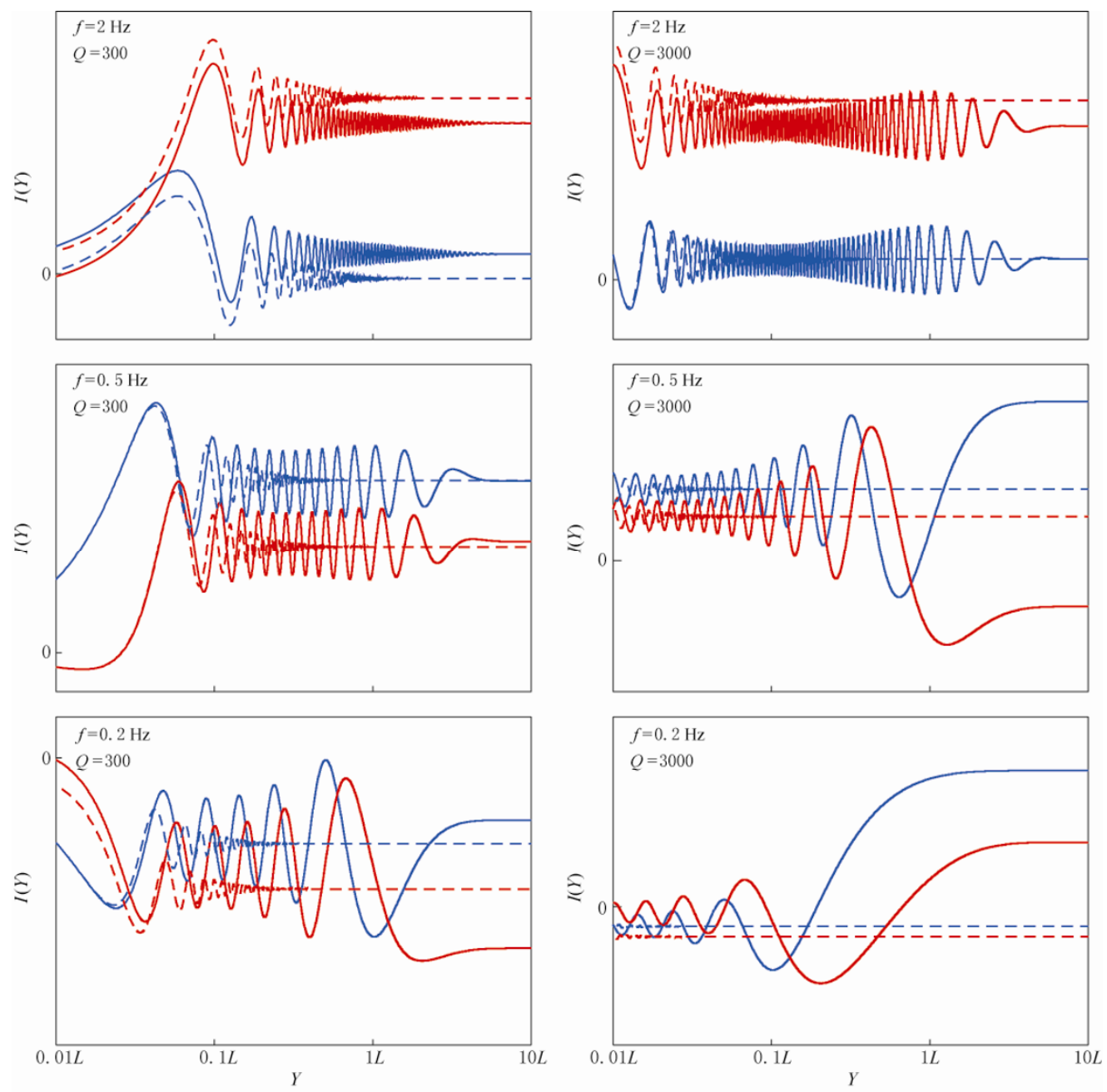

Figure 2 Integral $I(Y)$ defined by equation (7). In each panel, the solid lines and dashed lines are integrals $I(Y)$ for numerical computation and stationary phase approximation, respectively (red for real and blue for imaginary).

$$
\begin{gathered}
C(\omega)=\frac{2}{\pi k} \int_{\Sigma} \sqrt{\frac{r_{1}}{r_{2}}} \mathrm{e}^{\mathrm{i} k\left(r_{2}-r_{1}\right)} \mathrm{e}^{-\left(r_{1}+r_{2}\right) / 2 L}|S(\omega)|^{2} n \mathrm{~d} r_{1} \mathrm{~d} \theta= \\
\frac{2}{\pi k} \int_{0}^{\infty} \int_{\theta_{0}}^{2 \pi-\theta_{0}} \sqrt{\frac{r_{1}}{r_{2}}} \mathrm{e}^{\mathrm{i} k\left(r_{2}-r_{1}\right)} \mathrm{e}^{-\left(r_{1}+r_{2}\right) / 2 L}|S(\omega)|^{2} n \mathrm{~d} r_{1} \mathrm{~d} \theta,
\end{gathered}
$$

from which we can see that all quantities in equation (5) that depend on $x$ and $y$ now depend on $r_{1}$ and $\theta$, which are shown in Figure 1. The cutoff $\theta_{0}$ is introduced to ensure that the integral is over the left half-plane.

$$
\theta_{0}=\left\{\begin{array}{lll}
0 & \text { for } & r_{1}<R / 2 \\
\arccos \left(R / 2 r_{1}\right) & \text { for } & r_{1} \geq R / 2
\end{array}\right.
$$

For brevity, we assume that the power spectrum of ambient noise is spatially constant. In accordance with Snieder (2004), we also assume that the scatterer density $n$ is constant in space. However, it is still difficult to deal with the infinite integral over $r_{1}$. Fortunately, the integrand's amplitude is controlled by $\exp \left[-\left(r_{1}+r_{2}\right) / 2 L\right]$, which attenuates exponentially as $r_{1}$ increases. So we can replace the upper limit of $r_{1}$ with a cutoff value, for example, $20 L$ ( $L$ is the attenuation length), for which the amplitude is only $2 \times 10^{-9}$ of its initial amplitude. $\mathrm{Nu}-$ merical tests show that larger cutoff values do not change the results. Then equation (8) could be simplified as

$$
\begin{gathered}
C(\omega)=\frac{2 n}{\pi k}|S(\omega)|^{2} \times \\
\int_{0}^{20 L} \int_{\theta_{0}}^{2 \pi-\theta_{0}} \sqrt{\frac{r_{1}}{r_{2}}} \mathrm{e}^{\mathrm{i} k\left(r_{2}-r_{1}\right)} \mathrm{e}^{-\left(r_{1}+r_{2}\right) / 2 L} \mathrm{~d} r_{1} \mathrm{~d} \theta .
\end{gathered}
$$

Now the double integral is ready to be computed numerically. We partition the integration intervals of both 
$r_{1}$ and $\theta$ into many small enough subintervals, apply the 6-point Gauss-Legendre integration on each subinterval, and sum the results up to get the final $C(\omega)$.

\section{Comparison between cross correla- tion and Green's function}

We assume the phase velocity $c$ to be $3.5 \mathrm{~km} / \mathrm{s}$, quality factor $Q$ to be 300 . Receiver distance $R$ is set to be $50 \mathrm{~km}, 100 \mathrm{~km}$ or $200 \mathrm{~km}$, which are three typical station distances in ambient seismic noise studies. For each $R$, we evaluate the approximate and numerical cross correlation function $C(\omega)$ (denoted by $C_{\mathrm{ap}}(\omega)$ and $C_{\mathrm{nu}}(\omega)$ in the following, for brevity) by equation (6) and equation (10), respectively, for frequency from $0.01 \mathrm{~Hz}$ to $1 \mathrm{~Hz}$, with step length of $0.001 \mathrm{~Hz}$. As $C_{\text {ap }}(\omega)$ and $C_{\mathrm{nu}}(\omega)$ are complex, we present the phase difference and amplitude difference, respectively.

3.1 Phase deviation $f_{\mathrm{ap}}(\omega)$ from $C_{\mathrm{nu}}(\omega)$ and its influence on phase velocity measurement

Figure 3 displays the phase difference $\delta \phi$ between $C_{\text {ap }}(\omega)$ and $C_{\text {nu }}(\omega)$, defined by

$$
\delta \phi=\phi_{\mathrm{ap}}-\phi_{\mathrm{nu}}=k R-\pi / 4-\phi_{\mathrm{nu}} .
$$

It should be noticed that the $x$ axis of Figure 3 is not frequency or angular frequency, but the number of wavelength between the two receivers, defined by

$$
N=\frac{R}{\lambda} .
$$

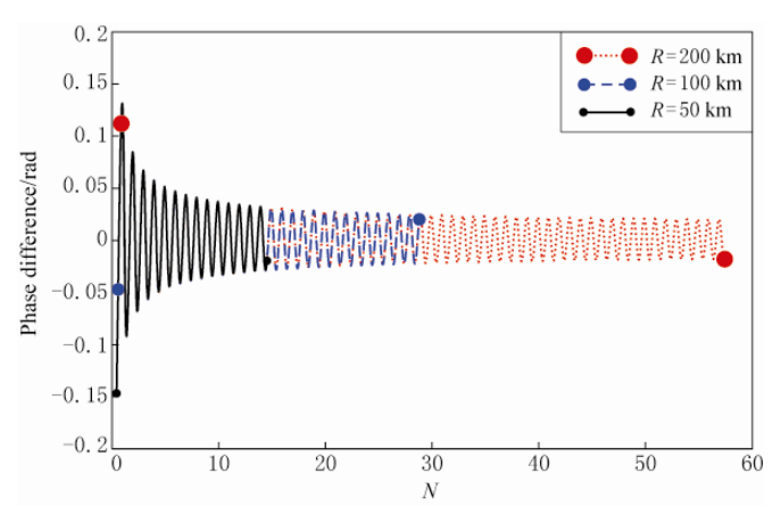

Figure 3 Phase difference between the approximate and the exact solution of cross correlation function $C(\omega)$. The vertical axis is phase difference (in radian) over $\pi$. Curves of different color are for different receiver distance $R$, as shown by the legend. $N$ is the number of wavelengths between the two stations, $R / \lambda$. The dots with different color and sizes denote the ends of the curves, respectively.
Curves of different color are for different receiver distance $R$ (black, blue, and red are for $R=50,100,200 \mathrm{~km}$, respectively). The dots with different colors and sizes denote the ends of the curves, respectively. The common parts of the three curves are identical, which is expected by the scale analysis. It is very obvious from Figure 3 that there is indeed phase difference between $C_{\text {ap }}(\omega)$ and $C_{\text {nu }}(\omega)$. The deviation oscillates with smaller amplitude as $N$ increases. When $N<1$, the deviation could exceed $\pi / 10$ and when $N$ is big enough $(N>30)$, the deviation will be below $\pi / 50$. Figure 4 is the zoomed view of the $N<10$ part in Figure 3. In many studies, only the station pairs whose distance is above several wavelengths are used, and then the phase difference is up to $\pi / 20$ according to Figure 4 .

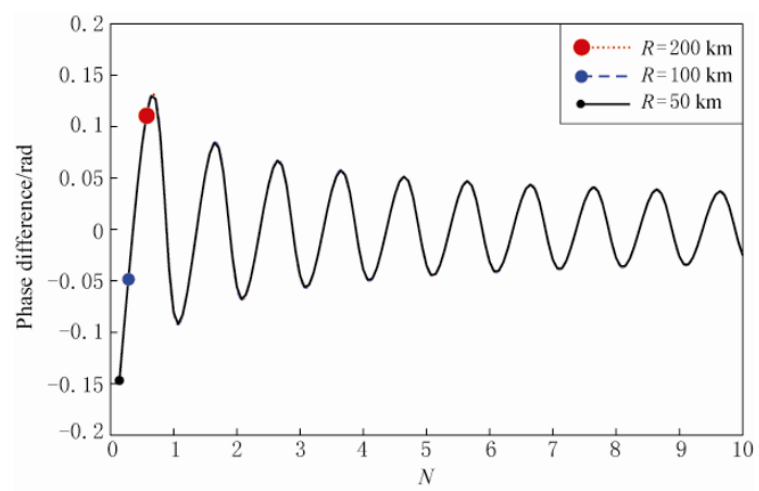

Figure 4 Zoomed view of the $N<10$ part in Figure 3.

It is straightforward to translate the phase deviation into errors of phase velocity. From equation (11) we know that the correct formula to measure the phase velocity is

$$
c=\frac{R}{t-T / 8+\delta \phi / \omega},
$$

where $T$ is the period, $t$ is the travel time of the surface wave. However, if $C_{\text {ap }}(\omega)$ is taken as exact Green's function, the formula of phase velocity measurement will be written as

$$
c^{\prime}=\frac{R}{t-T / 8},
$$

which is without the phase deviation $\delta \phi$. Then we define the relative error as

$$
\frac{\delta c}{c^{\prime}}=\frac{c^{\prime}-c}{c^{\prime}}=\frac{\delta \phi}{k R}=\frac{\delta \phi}{2 \pi N} .
$$

This means that the relative error introduced by the phase deviation decreases very fast as $N$ increases. The 
result of $\delta c / c^{\prime}$ is shown in Figure 5, also with the $x$ axis of $N<10$. When $N$ is small $(N<2)$, the relative error is substantial $(>3 \%)$. However, for the receiver distances used in most of seismological studies $(N>5)$, the relative error is less than $1 \%$.

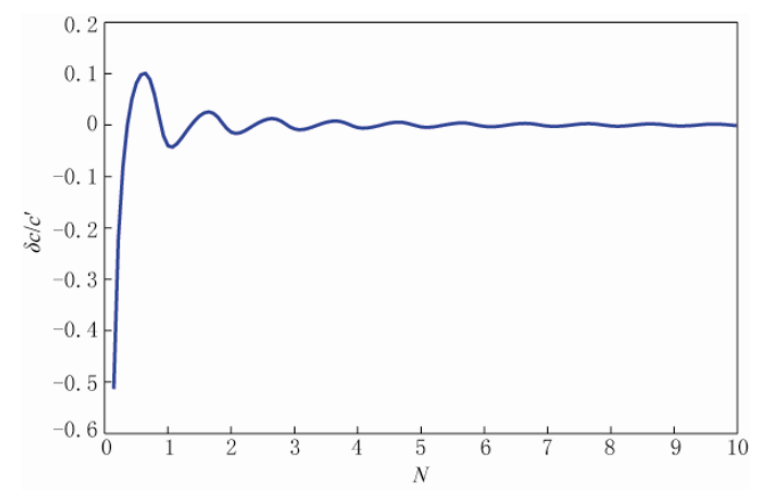

Figure 5 Relative error of phase velocity introduced by the approximation. $N$ is the number of wavelength between receivers, and the vertical axis is the ratio between phase velocity error and the true phase velocity.

\subsection{Amplitude deviation $C_{\mathrm{af}}(\omega)$ from $C_{\mathrm{nu}}(\omega)$}

The amplitude of Green's function (equation (1)) contains the information of geometrical spreading and attenuation of the medium. The amplitudes of $C_{\mathrm{ap}}(\omega)$ and $C_{\text {nu }}(\omega)$ (denoted as $A_{\text {ap }}$ and $A_{\text {nu }}$ respectively in the following) for different values of $R$ are shown in Figure 6. The red curves stand for approximate solutions and the blue ones for the numerical solutions. Although they agree with each other fairly well, the numerical amplitude oscillates around the approximate amplitude. To quantitatively present the amplitude differences between $C_{\text {ap }}(\omega)$ and $C_{\text {nu }}(\omega)$, we define the relative error of amplitude, just as what we do with the phase velocity.

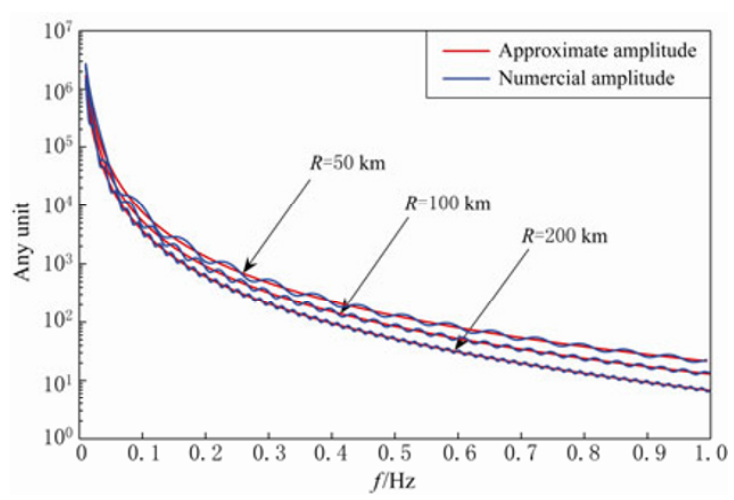

Figure 6 Amplitude of approximate and exact solution of cross correlation function $C(\omega)$.

$$
\frac{\delta A}{A_{\text {ap }}}=\frac{A_{\text {nu }}-A_{\text {ap }}}{A_{\text {ap }}}
$$

The result is shown in Figure 7. The $x$ axis is the number of wavelength between the two receivers, $A$ and $B$. The dots with different colors and sizes denote the ends of the curves, respectively. The relative error of amplitude has similar pattern with the phase difference shown in Figure 3 , which is oscillating around zero and with decreasing magnitude. When $N<3$, the mismatch is more than $10 \%$. But for $R$ used in most studies, the amplitude difference is less than $10 \%$.

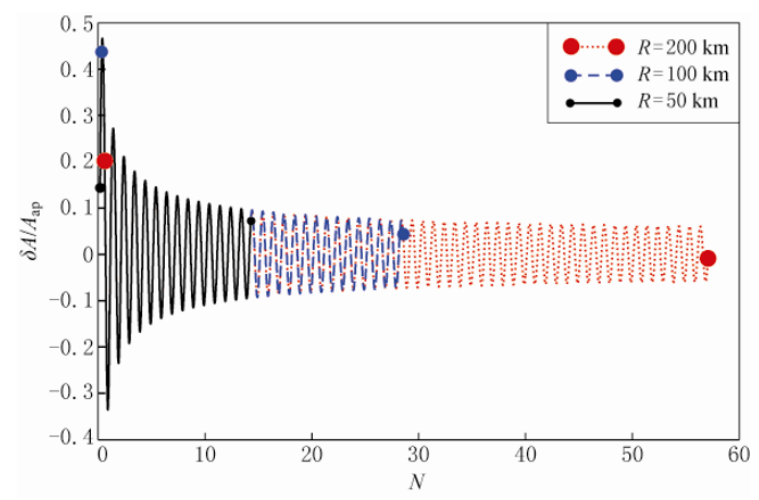

Figure 7 Relative difference of amplitude between the approximate and the exact solution of cross correlation function $C(\omega)$. The vertical axis is the ratio between amplitude error and the true amplitude. The dots with different colors and sizes denote the ends of the curves, respectively.

\section{Discussion and conclusions}

The theoretical framework (Snieder, 2004, 2007) is very helpful for understanding emergence of Green's function from cross correlation of ambient noise. His approach is more appropriate for application of ambient noise to seismology, as it neither require equipartition of energy, nor time-reversal invariance of wave equation in elastic medium, which seems to be absent for our Earth. However, a stationary phase approximation was introduced to deal with the integral equation (5) over the whole plane in the derivation, which seems to work well in high frequency band. To study the error resulted from the approximation, we numerically evaluated the integral equation (5) to get a more accurate result and compare it with the real Green's function. We find that the approximation is effective as the exact and approximate solution agree with each other generally. However, it indeed introduces some errors to equation (1). There is an oscillating deviation of both amplitude and phase in 
the approximate solution from the exact solution, which is around zero and attenuating when the frequency increases. This means that the stationary phase approximation is not appropriate when the frequency is relatively low (that is, the number of wavelength between receivers are very small), but suitable for the high frequency condition.

Although theoretically the relationship shown in equation (1) is not accurate, it does not affect most applications of ambient seismic noise method, as in almost all the researches only the station pairs whose distances are above several wavelengths are used to compute the cross correlation. Our computation implies that if the receiver distances are above several wavelengths, the error introduced by the approximation would be negligible. We recommend that only the station pairs whose distances are above 5 wavelengths can be used.

In this research, it is still assumed that the scattered waves propagate isotropically near the receivers. As we treat the scatterers as isotropic, mathematically this assumption is expressed by the condition that the scatterer density $n$ is constant in space. However, many practical researches found that this might not be the actual case. Sabra et al. (2005) found that the SNR of cross correlation function is higher for station-pairs oriented perpendicular to the coast line. Pederson and Kruger (2007) found that in Baltic shield the ambient seismic noise is very close to a plane wave in some frequency band. Some researchers even use this property to study the characteristic of ambient seismic noise (Stehly et al., 2006). All of these imply that it still need more theoretical and practical researches on the relationship between cross correlation function and the Green's function.

Acknowledgments This research was supported by the National Natural Science Foundation of China (No. 40674027) and CAS outstanding 100 research program, MOST program 2007FY220100.

\section{References}

Cho K H, Herrmann R B, Ammon C J and Lee K (2007). Imaging the upper crust of the Korean Peninsula by surface-wave tomography. Bull Seismol Soc Am 97(1): 198-207.

Cong L and Mitchell B J (1998). Seismic velocity and $Q$ structure of the middle eastern crust and upper mantle from surface wave dispersion and attenuation. Pure Appl Geophys 153(2-4): 503-538.

Derode A, Larose E, Campillo M and Fink M (2003a). How to estimate the Green's function of a heterogeneous medium between two passive sensors? Application to acoustic waves. Appl Phys Lett 83(15): 3 054-3 056.
Derode A, Larose E, Tanter, M, de Rosny J, Tourin A, Campillo $M$ and Fink M (2003b). Recovering the Green's function from field-field correlations in an open scattering medium (L). $J$ Acoust Soc Am 113(6): 2 973-2 976.

Draganov D, Campman X, Thorbecke J, Verdel A and Wapenaar K (2009). Reflection images from ambient seismic noise. Geophysics 74: A63-A67.

Kang T S and Shin J S (2006). Surface-wave tomography from ambient seismic noise of accelerograph networks in southern Korea. Geophys Res Lett 33(17): L17303.

Pedersen H A and Kruger F (2007). Influence of the seismic noise characteristics on noise correlations in the baltic shield. Geophys J Int 168(1): 197-210.

Roux P, Sabra K G, Gerstoft P, Kuperman W A and Fehler M C (2005). P-waves from cross-correlation of seismic noise. Geophys Res Lett 32(19): L19303.

Sabra K G, Gerstoft P, Roux P, Kuperman W A and Fehler M C (2005). Surface wave tomography from microseisms in Southern California. Geophys Res Lett 32(14): L14311.

Shapiro N M and Campillo M (2004). Emergence of broadband Rayleigh waves from correlations of the ambient seismic noise. Geophys Res Lett 31(7): L07614.

Shapiro N M, Campillo M, Stehly L and Ritzwoller M H (2005). High-resolution surface-wave tomography from ambient seismic noise. Science 307(5715): 1 615-1 618.

Snieder R (2004). Extracting the Green's function from the correlation of coda waves: A derivation based on stationary phase. Phys Rev E 69(4): 046610.

Snieder R (2007). Extracting the Green's function of attenuating heterogeneous acoustic media from uncorrelated waves. $J$ Acoust Soc Am 121(5): 2 637-2 643.

Stehly L, Campillo M and Shapiro N M (2006). A study of the seismic noise from its long-range correlation properties. $J$ Geophys Res 111(B10): B10306.

Villasenor A, Yang Y, Ritzwoller M H and Gallart J (2007). Ambient noise surface wave tomography of the Iberian Peninsula: Implications for shallow seismic structure. Geophys Res Lett 34(11): L11304.

Weaver R L and Lobkis O I (2001). Ultrasonics without a source: Thermal fluctuation correlations at $\mathrm{MHz}$ frequencies. Phys Rev Lett 87(13): 134301.

Weaver R L and Lobkis O I (2002). On the emergence of the Green's function in the correlations of a diffuse field: pulse-echo using thermal phonons. Ultrasonics 40(1-8): 435-439.

Yang Y, Ritzwoller M H, Levshin A L and Shapiro N M (2006). Ambient noise Rayleigh wave tomography across Europe. Geophys J Int 168(1): 259-274.

Yao H, van der Hilst R D and de Hoop M V (2006). Surface-wave array tomography in SE Tibet from ambient seismic noise and two-station analysis - I. Phase velocity maps. Geophys J Int 166(2): 732-744.

Zhan Z, Ni S, Helmberger D and Clayton R (2010). Retrieval of Moho-reflected shear wave arrivals from ambient seismic noise. Geophys J Int 182(1): 408-420. 